\title{
Carbono orgânico e biomassa microbiana do solo em plantios de Acacia mangium no Cerrado de Roraima
}

\author{
Sara Magda Oliveira SIMÕES ${ }^{1}$, Jerri Édson ZILLI², Mirian Cristina Gomes COSTA³, Hélio TONINI ${ }^{4}$, \\ Fabiano de Carvalho BALIEIRO5
}

\section{RESUMO}

O objetivo do estudo foi avaliar os efeitos de plantios de Acacia mangium, localizados no cerrado em Roraima, sobre o carbono orgânico e biomassa microbiana do solo. Foram realizadas amostragens de solo nas profundidades de $0-20 \mathrm{~cm}$ e $20-40 \mathrm{~cm}$ em dois plantios de $A$. mangium com cerca de cinco anos de idade, e em duas áreas de Cerrado nativo consideradas referência. Um dos plantios de $A$. mangium (localizado na Fazenda Cigolina) correspondeu a um plantio homogêneo (espaçamento de 3,6 m entre linhas e 2,0 m entre plantas) enquanto que o outro (localizado no Campo Experimental Água Boa - CEAB) correspondeu a um plantio em faixas com duas linhas de plantio (espaçamento de $6 \mathrm{~m}$ entre linhas, 2,5 m entre plantas e cerca de $30 \mathrm{~m}$ entre faixas). As amostras de solo foram analisadas quanto ao carbono orgânico, carbono da biomassa microbiana, respiração basal do solo e quociente metabólico, além de atributos químicos de fertilidade. Foi verificado que os plantios de A. mangium não proporcionaram aumentos significativos do carbono orgânico do solo em comparação às áreas de referência. Entretanto, na média geral, esses plantios proporcionaram aumento do carbono da biomassa microbiana do solo e reduçáo do quociente metabólico, indicando a possibilidade de acúmulo de carbono orgânico no solo em longo prazo. Também foi observado que, em comparaçáo ao plantio da fazenda Cigolina e às áreas de referência, o carbono microbiano do solo foi maior e acompanhado de menor quociente metabólico no plantio de $A$. mangium no $\mathrm{CEAB}$, mostrando que a estrutura de plantio exerceu influência sobre a biomassa microbiana do solo.

PALAVRAS-CHAVE: Respiração basal do solo, quociente metabólico, Amazônia, plantios florestais, qualidade do solo.

\section{Soil organic carbon and soil microbial biomass in Acacia mangium plantation in the Savanna of Roraima}

\begin{abstract}
The aim of this study was to evaluate the effects of Acacia mangium plantation in the Roraima's Savanna, on soil organic carbon and soil microbial biomass. Soil samplings were collected on the depths of $0-20 \mathrm{~cm}$ and $20-40 \mathrm{~cm}$ in two Acacia mangium plantation sites, about five years old, and in two sites of native savanna as reference. One of the $A$. mangium sites (located at Fazenda Cigolina) was composed by a homogeneous plantation $(3.6 \mathrm{~m}$ between row and $2.0 \mathrm{~m}$ between trees) and, the other one (located at Campo Experimental Agua Boa Experimental - CEAB) was composed by a plantation on which the planting distances had strips of two rows 6 meters apart, 2.5 meters between trees and 30 meters between strips). Soil samples were analyzed regarding soil organic carbon, microbial biomass carbon, soil basal respiration and metabolic quotient, besides soil fertility attributes. Results showed that $A$. mangium plantations did not provide significant increases of organic carbon on the soil comparing with sites used as reference. However, generally, $A$. mangium plantation increased soil microbial biomass carbon and reduced metabolic quotient, indicating a possibility of soil organic carbon accumulation in long-term. It was also observed that, soil biomass microbial carbon was higher, followed by smaller metabolic quotient at CEAB A. mangium plantation compared with Cigolina and reference sites, showing that planting design exercised influence on soil microbial biomass.
\end{abstract}

KEYWORDS: Soil basal respiration, metabolic quotient, Amazonian, forest plantation, soil quality.

\footnotetext{
1 Universidade Federal de Roraima, UFRR, e-mail: saramagda18@hotmail.com

2 Empresa Brasileira de Pesquisa Agropecuária, Embrapa Roraima, e-mail: zilli@cpafrr.embrapa.br

${ }^{3}$ Universidade Federal do Ceará, e-mail: mirian.costa@ufc.br

${ }^{4}$ Empresa Brasileira de Pesquisa Agropecuária, Embrapa Roraima, e-mail: helio@cpafrr.embrapa.br

${ }^{5}$ Empresa Brasileira de Pesquisa Agropecuária, Embrapa Solos, e-mail: balieiro@cnps.embrapa.br
} 


\section{INTRODUÇÃO}

A Acacia mangium (acácia) é uma espécie leguminosa nativa da Austrália e Papua Nova Guiné que tem sido plantada em diferentes regióes do planeta para diversos usos. Por ser de rápido crescimento (Ghosh \& Verma, 2006), apresentar baixa exigência nutricional (Dias et al., 1994), aportar quantidades expressivas de nutrientes ao solo via serapilheira (Andrade et al., 2000) e estabelecer simbiose com bactérias fixadoras de nitrogênio e fungos microrrízicos (Sun et al., 1992; Ghosh \& Verma, 2006), essa espécie tem sido bastante utilizada em programas de recuperação de áreas degradadas em diversos países, inclusive no Brasil (Franco \& Faria, 1997; Duguma et al., 1994; Garay et al., 2003). Por outro lado, plantios comerciais dessa essência florestal com fins de produção de celulose e madeira são comuns na Ásia, especialmente na Indonésia (N.R.C, 1983) e ainda pouco expressiva no Brasil, embora haja aptidão edafoclimática favorável (Andrade $e t$ al., 2000).

Roraima apresenta grande área de Cerrado, no qual o solo possui baixa fertilidade natural, baixo teor de matéria orgânica e reduzida capacidade de retenção de água (Melo et al., 2004). Entretanto, embora estas características possam representar limitaçôes para culturas mais exigentes, ao cultivo da Acacia mangium essas áreas têm mostrado aptidão. Desta forma, a partir do final dos anos 90 do século passado, foram estabelecidos plantios de acácia no Cerrado de Roraima com o objetivo de aproveitamento para madeira e celulose. Em função dos resultados iniciais animadores e investimentos estrangeiros, os plantios expandiram-se significativamente, despertando a preocupaçáo quanto ao impacto ambiental da conversão do lavrado em plantios florestais homogêneos. Essas preocupaçôes vão desde o possível impacto sobre os recursos hídricos e da biodiversidade, até as alteraçóes nos estoques de carbono e na qualidade do solo.

A introdução de cultivos anuais ou perenes em ecossistemas naturais altera os fluxos de energia e nutrientes no sistema, com conseqüências muitas vezes negativas aos estoques de carbono do solo e na biodiversidade (Luizão et al., 1992; Corazza et al., 1999; Sposito \& Zabel, 2003; Remigi et al., 2008).

Estudos conduzidos em regiôes tropicais têm mostrado que a introdução da Acacia mangium e de outras espécies vegetais de rápido crescimento, pode reduzir e alterar a composição, funcionalidade e a diversidade da comunidade microbiana do solo (Andrade, 1999; Remigi et al., 2008), tornando imprescindível a avaliação dos impactos da introdução desta espécie exótica sobre os diferentes componentes do ecossistema.

Em solos intemperizados, como os encontrados no Cerrado de Roraima, a dinâmica da matéria orgânica é extremamente importante, influenciando na fertilidade e qualidade do solo e, conseqüentemente, em sua capacidade produtiva. Esta dinâmica é regida por diversos fatores e caracterizada por transformaçôes que dependem da biomassa microbiana do solo e resulta na disponibilidade ou indisponibilidade de nutriente às plantas (De-Polli \& Guerra, 1999).

O estoque de $\mathrm{C}$ do solo compreende fraçóes intimamente associadas aos minerais, até fraçôes mais lábeis, pouco ou não associadas à fração mineral, como os resíduos vegetais existentes entre e dentro de agregados do solo (Roscoe \& Machado, 2002). O C associado à biomassa microbiana é o componente mais ativo da fração lábil, pois transforma e transfere energia e nutrientes para os demais componentes do ecossistema, sendo atualmente usado, conjuntamente com outros atributos, como indicador da qualidade ambiental e da sustentabilidade de agroecossistemas (De-Polli \& Guerra, 1999; Haynes et al., 2000).

Tanto o C carbono orgânico do solo quanto o C da biomassa microbiana têm sido utilizados como indicadores de alteraçôes e de qualidade do solo, uma vez que estáo associados às funçôes ecológicas do ambiente e são capazes de refletir as mudanças de uso do solo (Jackson et al., 2003).

Neste sentido, este trabalho objetivou avaliar os efeitos de plantios de Acacia mangium, localizados no Cerrado do Estado de Roraima, sobre o carbono orgânico e biomassa microbiana do solo.

\section{MATERIAL E MÉTODOS}

\section{DESCRIÇÃO DOS LOCAIS DE COLETA E AMOSTRAGEM}

No mês de agosto de 2006 foram coletadas amostras de solo no Campo Experimental Água Boa (CEAB) da Embrapa Roraima (latitude $2^{\circ} 39^{\prime} 48^{\prime \prime} \mathrm{N}$ e longitude $60^{\circ} 50^{\prime} 15^{\prime \prime} \mathrm{W}$ ) e na fazenda Cigolina (núcleo Jacitara) da empresa Ouro Verde Agrosilvopastoril Ltda. (latitude $2^{\circ} 7{ }^{\prime} 19^{\prime \prime} \mathrm{N}$ e longitude $60^{\circ} 46^{\prime}$ 6" W), ambos locais no município de Boa Vista. O clima da região, de acordo com a classificação de Köppen, é do tipo Aw, tropical chuvoso, com precipitação média anual de, aproximadamente, $1600 \mathrm{~mm}$ e umidade relativa do ar em torno de $70 \%$.

No CEAB foi amostrado o solo em um plantio de acácia, implantado em um Latossolo Amarelo há cerca de 5 anos e, também, em uma área de referência não cultivada no Cerrado, denominada de Cerrado nativo (CN). Anteriormente, o plantio de acácia em questão fez parte de um experimento para estudo de sistema agroflorestal, cujo plantio das mudas foi realizado em seqüência da cultura da soja. A estrutura de plantio das acácias corresponde à disposiçáo das plantas em faixas com duas linhas de plantio (espaçamento de $6 \mathrm{~m}$ entre linhas e $2,5 \mathrm{~m}$ entre plantas e cerca de $30 \mathrm{~m}$ entre faixas), tendo sido a amostragem realizada entre as linhas de plantio. Antes do plantio da soja havia sido realizada a aplicação de $600 \mathrm{~kg} \mathrm{ha}^{-1}$ de calcário e $230 \mathrm{~kg} \mathrm{ha}^{-1}$ da fórmula 10-26-26 e, 
no plantio das acácias foram aplicados $100 \mathrm{~g}$ de calcário, 3 litros de esterco de gado, $250 \mathrm{~g}$ da fórmula 10-26-26 e 150 $\mathrm{g}$ de uréia por cova de plantio.

$\mathrm{Na}$ fazenda Cigolina foi amostrado o solo em um plantio homogêneo de acácia (espaçamento de 3,6 m entre linhas e 2,0 $\mathrm{m}$ entre plantas), implantado também em Latossolo Amarelo há cerca de 5 anos, e uma área de referência no Cerrado ainda não cultivado, denominado também de $\mathrm{CN}$, o qual apresentava sinais de pastejo bovino esporádico. No plantio de acácia neste local a amostragem foi realizada tanto na entrelinha, quanto entre as plantas, isto porque, por ocasião da implantaçâo da lavoura, havia sido feita gradagem com o objetivo de formar camalháo para o plantio das mudas. Neste local, a adubação de plantio foi constituída por $500 \mathrm{~kg} \mathrm{ha}^{-1}$ de calcário aplicado na linha de plantio e aplicação de $100 \mathrm{~g}$ da fórmula 4-28-20 por cova de plantio.

Em cada um dos plantios de acácia e também no $\mathrm{CN}$ foram coletadas três amostras compostas por dez amostras simples de solo. As amostras foram coletadas com auxílio de trado tipo holandês, nas profundidades de 0-20 e $20-40 \mathrm{~cm}$. As amostras foram analisadas quanto à fertilidade, textura e carbono orgânico do solo (Embrapa, 1997), e quanto ao carbono da biomassa microbiana do solo (C-BMS), respiração basal do solo (RBS) e quociente metabólico ( $q \mathrm{CO} 2)$.

\section{DETERMINAÇÃO DO C-BMS, RBS E QCO2.}

A determinação do carbono da biomassa microbiana do solo (C-BMS) foi realizada pelo método de fumigação e extração (Vance et al. 1987), com o uso de $20 \mathrm{~g}$ de solo, fumigaçáo com $1,0 \mathrm{~mL} \mathrm{CHCl}$ aplicado diretamente sobre o solo da amostra (Silva et al., 2007; De-Polli \& Guerra, 2008) e extração com $50 \mathrm{~mL}$ de $\mathrm{K}_{2} \mathrm{SO}_{4}$ a $0,5 \mathrm{~mol} \mathrm{~L}^{-1}$. O cálculo do

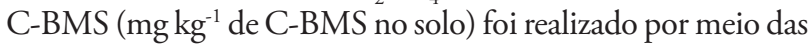
fórmulas: $\mathrm{C}-\mathrm{BMS}=\mathrm{FC} \mathrm{kc}^{-1}$, em que FC = CSF-CSNF, sendo CSF o teor de C extraído do solo fumigado, CSNF o teor de $\mathrm{C}$ extraído do solo não fumigado e kc - fator de correção (utilizado valor de 0,33). O CSF ou CSNF $=(\mathrm{Vb}-\mathrm{Va}) \mathrm{CSFA}$ $0,00350(8 \mathrm{Ms})^{-1}$, em que $\mathrm{Vb}$ - volume $(\mathrm{mL})$ de $\left(\mathrm{NH}_{4}\right)_{2}$ $\mathrm{Fe}\left(\mathrm{SO}_{4}\right)_{2} \cdot 6 \mathrm{H}_{2} \mathrm{O}$ (SFA) gasto na titulação da solução do ensaio em branco; $\mathrm{Va}$ - volume $(\mathrm{mL})$ de $\left(\mathrm{NH}_{4}\right)_{2} \mathrm{Fe}\left(\mathrm{SO}_{4}\right)_{2} \cdot 6 \mathrm{H}_{2} \mathrm{O}$ (SFA) gasto na titulação da solução do ensaio com a amostra; CSFA = concentração do SFA $\left(\mathrm{mol} \mathrm{L}^{-1}\right)$ e, Ms= massa da amostra de solo seco $(\mathrm{g})$. Para estimar a respiração basal do solo (RBS), seguiu-se o procedimento descrito por Jenkinson \& Powlson, (1976), utilizando-se fraçóes de $50 \mathrm{~g}$ de solo incubadas hermeticamente em frascos de vidro na presença de $10 \mathrm{~mL}$ de $\mathrm{NaOH}$ a $0,5 \mathrm{~mol} \mathrm{~L}^{-1}$ por 5 dias. O cálculo da RBS ( $\mu \mathrm{g} \mathrm{kg}^{-1} \mathrm{~h}^{-1} \mathrm{C}-\mathrm{CO}_{2}$ no solo) foi realizado pela fórmula: RBS $=\mathrm{Vb}-\mathrm{Va} \mathrm{CHCl} 61000 \mathrm{Ms}^{-1} \mathrm{~T}^{-1}$, em que, $\mathrm{Vb}$ - volume de $\mathrm{HCl}(\mathrm{mL})$ gasto na titulaçáo do $\mathrm{NaOH}$ do ensaio em branco; $\mathrm{Va}$ - volume de $\mathrm{HCl}(\mathrm{mL})$ gasto na titulaçáo do $\mathrm{NaOH}$ do ensaio com a amostra, $\mathrm{CHCl}$, concentraçáo do $\mathrm{HCl}$ (mol
$\left.\mathrm{L}^{-1}\right), \mathrm{Ms}$ - massa de solo incubado seco (g); e T - tempo de incubação (h). O quociente metabólico $\left(q \mathrm{CO}_{2}\right)\left(\mu \mathrm{g} \mathrm{g}^{-1} \mathrm{~h}^{-1}\right.$ de $\mathrm{C}-\mathrm{CO}_{2}$ do C-BMS), por sua vez, foi estimado pela razão entre a respiração basal do solo e o $\mathrm{C}$ da biomassa microbiana (Anderson \& Domsch, 1990), seguindo a fórmula: $q \mathrm{CO}_{2}=$ (RBS)/C-BMS.

\section{ANÁLISE ESTATÍSTICA}

Os dados foram analisados estatisticamente em esquema fatorial $2 \times 2 \times 2$ - dois locais (CEAB e fazenda Cigolina), duas coberturas do solo (plantio de acácia e $\mathrm{CN}$ ) e duas profundidades de amostragem $(0-20 \mathrm{~cm}$ e $20-40 \mathrm{~cm})$ - por meio de análise da variância pelo teste $\mathrm{F}$ e Tukey em 5\% de significância.

\section{RESULTADOS E DISCUSSÃO}

A partir da análise granulométrica do solo foi verificado que ambos os locais de estudo apresentam solos com textura arenosa (Embrapa, 2006). De modo geral, os valores de $\mathrm{pH}$, cálcio $(\mathrm{Ca})$, magnésio $(\mathrm{Mg})$, potássio $(\mathrm{K})$ e fósforo $(\mathrm{P})$ foram baixos ou muito baixos, conforme as tabelas de interpretação de análise de solos de Cerrado (Sousa \& Lobato, 2004). Tal interpretação mostra a natureza distrófica dos solos do Cerrado Roraimense, típica de Latossolos desse bioma (Lopes, 1984; Melo et al., 2004).

No $\mathrm{CN}$, à exceção dos elementos $\mathrm{Ca}$ e $\mathrm{Mg}$, cujos teores foram significativamente maiores na fazenda Cigolina, tanto nos primeiros $20 \mathrm{~cm}$ de profundidade, quanto $20-40 \mathrm{~cm}$, não foram observadas diferenças significativas nos demais atributos químicos do solo (Tabela 1). Os maiores teores de $\mathrm{Ca}$ e $\mathrm{Mg}$ encontrados no $\mathrm{CN}$ da fazenda Cigolina indicam a possibilidade de ter sido realizada calagem em anos anteriores em virtude do possível uso da área para pastejo bovino, ou então uma variação natural do solo.

$\mathrm{Na}$ fazenda Cigolina foram observados valores de Ca significativamente maiores no solo do plantio de acácia em comparação ao $\mathrm{CN}$ na profundidade de $0-20 \mathrm{~cm}$, o que náo foi observado no CEAB. Na fazenda Cigolina os valores de cálcio e magnésio no plantio de acácia foram maiores em relação ao observado no CEAB (Tabela 1). Estes teores de Ca trocável do solo em ambas as áreas de plantio de acácia, embora baixos, não devem limitar o crescimento da acácia dada à baixa exigência por esse elemento na fase inicial de desenvolvimento (Dias et al., 1994) e devido à boa performance que a espécie tem apresentado em áreas degradadas (Franco et al., 1994; Campello, 1998; Chada et al., 2004). Já os baixos teores de $\mathrm{Mg}, \mathrm{P}$ e K podem causar restriçóes ao desenvolvimento dessa essência tendo em vista que estáo abaixo dos níveis críticos para várias espécies florestais (Neves et al., 1990; Dias et al., 1994; Balieiro et al., 2004). 
Tabela 1 - Resultado da análise de fertilidade e granulometria do solo em áreas de plantio de Acacia mangium (Acácia) e Cerrado nativo de referência (CN) em Roraima.

\begin{tabular}{|c|c|c|c|c|c|c|c|c|c|c|c|}
\hline \multirow{2}{*}{ Profundidade } & \multirow{2}{*}{ Local } & \multirow{2}{*}{ Cobertura } & \multirow{2}{*}{$\begin{array}{c}\mathrm{pH} \\
\left(\mathrm{H}_{2} \mathrm{O}\right)\end{array}$} & $\mathrm{Al}$ & $\mathrm{Ca}$ & $\mathrm{Mg}$ & K & \multirow{2}{*}{$\frac{P}{\mathrm{mg} \mathrm{dm}^{-3}}$} & Areia & \multirow{2}{*}{$\frac{\text { Silte }}{\mathrm{g} \mathrm{kg}^{-1}}$} & \multirow[t]{2}{*}{ Argila } \\
\hline & & & & \multicolumn{4}{|c|}{$\mathrm{cmol}_{\mathrm{c}} \mathrm{dm}^{-3}$} & & & & \\
\hline \multirow{4}{*}{$0-20 \mathrm{~cm}$} & \multirow{2}{*}{ CEAB } & Acácia & $4,5 \mathrm{aA}$ & $0,49 \mathrm{aA}$ & $0,35 \mathrm{aB}$ & $0,04 a B$ & $0,03 \mathrm{aA}$ & $3,75 \mathrm{aA}$ & $852 \mathrm{aA}$ & $15 \mathrm{aB}$ & $133 \mathrm{aA}$ \\
\hline & & $\mathrm{CN}$ & $4,5 a A$ & $0,49 \mathrm{aA}$ & $0,31 \mathrm{aB}$ & $0,01 \mathrm{aB}$ & $0,01 \mathrm{bA}$ & $1,27 \mathrm{bA}$ & $844 a A$ & $21 \mathrm{aB}$ & $135 \mathrm{aA}$ \\
\hline & \multirow{2}{*}{ Cigolina } & Acácia & $4,5 a A$ & $0,49 \mathrm{aA}$ & $1,66 a A$ & $0,22 \mathrm{aA}$ & $0,00 \mathrm{aB}$ & $4,09 \mathrm{aA}$ & $681 \mathrm{aB}$ & $198 a A$ & $126 \mathrm{aA}$ \\
\hline & & $\mathrm{CN}$ & $4,8 \mathrm{aA}$ & $0,48 \mathrm{aA}$ & $1,37 \mathrm{bA}$ & $0,23 \mathrm{aA}$ & $0,00 \mathrm{aA}$ & $2,52 \mathrm{bA}$ & $710 \mathrm{aB}$ & $160 \mathrm{aA}$ & $130 \mathrm{aA}$ \\
\hline \multirow{4}{*}{$20-40 \mathrm{~cm}$} & \multirow{2}{*}{ CEAB } & Acácia & $4,5 a A$ & $0,50 \mathrm{aA}$ & $0,40 \mathrm{aB}$ & $0,01 \mathrm{aB}$ & $0,01 \mathrm{aA}$ & $2,02 \mathrm{aA}$ & 784aA & $30 \mathrm{aB}$ & $186 \mathrm{aA}$ \\
\hline & & $\mathrm{CN}$ & $4,5 a A$ & $0,46 \mathrm{aA}$ & $0,40 \mathrm{aB}$ & $0,01 \mathrm{aB}$ & $0,01 \mathrm{aA}$ & $0,81 \mathrm{bA}$ & $805 a A$ & $18 \mathrm{aB}$ & $177 \mathrm{aA}$ \\
\hline & \multirow{2}{*}{ Cigolina } & Acácia & $4,7 \mathrm{aA}$ & $0,42 \mathrm{aA}$ & $1,25 \mathrm{aA}$ & $0,18 \mathrm{aA}$ & $0,00 \mathrm{aA}$ & $3,93 \mathrm{aA}$ & $673 a B$ & $176 a A$ & $151 \mathrm{aA}$ \\
\hline & & $\mathrm{CN}$ & $4,5 \mathrm{bA}$ & $0,38 \mathrm{aA}$ & $1,54 \mathrm{aA}$ & $0,22 \mathrm{aA}$ & $0,00 \mathrm{aA}$ & $1,52 \mathrm{bA}$ & $664 a A B$ & $160 \mathrm{aA}$ & $176 \mathrm{aA}$ \\
\hline CV (\%) & & & 0,85 & 5,96 & 3,99 & 34,65 & 41,14 & 34,59 & 3,32 & 3,95 & 4,94 \\
\hline
\end{tabular}

* Médias seguidas das mesmas letras minúsculas na mesma coluna, profundidade e local, não diferem entre si pelo teste de Tukey em $5 \%$ de probabilidade;

** Médias seguidas das mesmas letras maiúsculas na mesma coluna, profundidade e cobertura, não diferem entre si pelo teste Tukey a $5 \%$ de probabilidade;

Ainda quanto à análise química do solo, foi observado náo haver diferenças significativas no $\mathrm{pH}$ e nos teores de $\mathrm{Al}$, comparando-se os plantios de acácia com o $\mathrm{CN}$, nos dois locais e profundidades (Tabela 1), embora hajam relatos na literatura que Acacia mangium, devido à grande extrusão radicular de $\mathrm{H}^{+}$em virtude da elevada absorção de cátions, tenha a capacidade de acidificar o solo (Binkley \& Giardina, 1997; Yamashita et al., 2008).

Em relação ao carbono orgânico $\left(\mathrm{C}_{\text {org }}\right)$ não foram observadas diferenças no CN entre CEAB e fazenda Cigolina, tendo sido encontradas médias de 7,62 e 5,45 g $\mathrm{kg}^{-1}$, respectivamente, nas profundidades de $0-20 \mathrm{~cm}$ e 20 $40 \mathrm{~cm}$ (Tabela 2). Estes valores de $\mathrm{C}_{\text {org }}$ situam-se numa faixa observada para áreas de savanas da Amazônia por Desjardins et al. (1996), sendo relativamente baixos quando comparados a solos de áreas de florestas e transição floresta-savana (Luizão \& Luizáo, 1997). Estes menores teores de $\mathrm{C}_{\text {org }}$ nas savanas geralmente ocorrem pela limitação de acúmulo de matéria orgânica no solo, devido ao menor aporte de liteira, à alta taxa de decomposição e, ainda, à textura arenosa do solo, como observado neste estudo (Tabela 1).

De forma semelhante, nos plantios de acácia, foram observados teores de $\mathrm{C}_{\text {org }}$ significativamente maiores nos primeiros $20 \mathrm{~cm}$ em ambos os locais do estudo. Entretanto, não foram observadas diferenças entre as coberturas nos dois locais e profundidades (Tabela 2).

A $A$. mangium é reconhecidamente uma espécie que aporta grande quantidade de liteira ao solo, principalmente por meio da queda de folhas, estruturas reprodutivas e ramos. Estudos conduzidos no Brasil e também na África, mostraram a entrada de cerca de 9-10 $\mathrm{Mg} \mathrm{ha}^{-1}$ ano $^{-1}$ de serapilheira em plantios de A. mangium ainda nos primeiros anos após a implantação (Bernhard-Reversat, 1993; Andrade etal., 2000; Garay et al., 2003). Este alto aporte de liteira associado a entrada de $\mathrm{N}$ biologicamente tende a aumentar o carbono orgânico do solo com o passar dos anos. Em solos arenosos das savanas africanas foi observado aumento significativo nos teores de carbono orgânico na interface liteira-solo, comparativamente com a

Tabela 2 - Carbono orgânico $\left(\mathrm{C}_{\text {org }}\right)$, carbono da biomassa microbiana do solo (C-BMS), respiração basal do solo (RBS), quociente metabólico $\left(q \mathrm{CO}_{2}\right)$ e relação C-BMS: $\mathrm{C}_{\text {org }}$ em amostras de solo de plantios de Acacia mangium (Acácia) e Cerrado nativo de referência (CN) em Roraima.

\begin{tabular}{|c|c|c|c|c|c|c|c|}
\hline Profundidade & Local & Cobertura & $\begin{array}{c}\mathrm{C}_{\text {org }} \\
\mathrm{g} \mathrm{kg}^{-1}\end{array}$ & $\begin{array}{l}\text { C-BMS } \\
\mathrm{mg} \mathrm{kg}^{-1}\end{array}$ & $\begin{array}{c}\text { RBS } \\
\mu \mathrm{g} \mathrm{kg}^{-1} \mathrm{~h}^{-1} \mathrm{C}-\mathrm{CO}_{2}\end{array}$ & $\begin{array}{c}q \mathrm{CO}_{2} \\
\mu \mathrm{g} \mathrm{g}^{-1} \mathrm{~h}^{-1} \text { de } \mathrm{C}-\mathrm{CO}_{2} \text { do } \\
\text { C-BMS }\end{array}$ & $\begin{array}{c}\text { C-BMS: } C_{\text {org }} \\
\%\end{array}$ \\
\hline \multirow{4}{*}{$0-20 \mathrm{~cm}$} & \multirow{2}{*}{ CEAB } & Acácia & $8,2 \mathrm{aA}$ & $322,3 \mathrm{aA}$ & $380,0 \mathrm{aA}$ & $1,2 \mathrm{bB}$ & $3,9 \mathrm{aA}$ \\
\hline & & $\mathrm{CN}$ & $8,0 \mathrm{aA}$ & $191,6 \mathrm{bA}$ & $330,0 \mathrm{bA}$ & $1,7 \mathrm{aA}$ & $2,4 \mathrm{bA}$ \\
\hline & \multirow{2}{*}{ Cigolina } & Acácia & $7,3 \mathrm{aA}$ & $171,9 \mathrm{aB}$ & $360,0 \mathrm{aA}$ & $2,1 \mathrm{aA}$ & $2,3 a B$ \\
\hline & & $\mathrm{CN}$ & $7,2 \mathrm{aA}$ & $152,0 \mathrm{aA}$ & $330,0 \mathrm{aA}$ & $2,2 \mathrm{aA}$ & $2,1 \mathrm{aA}$ \\
\hline \multirow{4}{*}{$20-40 \mathrm{~cm}$} & \multirow{2}{*}{ CEAB } & Acácia & $6,8 \mathrm{aA}$ & $192,9 \mathrm{aA}$ & $420,0 \mathrm{aA}$ & $2,2 \mathrm{bB}$ & $2,8 \mathrm{aA}$ \\
\hline & & $\mathrm{CN}$ & $5,7 \mathrm{aA}$ & $96,0 \mathrm{bA}$ & $330,0 \mathrm{~A}$ & $3,4 \mathrm{aA}$ & $1,7 \mathrm{bA}$ \\
\hline & \multirow{2}{*}{ Cigolina } & Acácia & $5,7 a A$ & $69,1 \mathrm{aB}$ & $270,0 \mathrm{aB}$ & $3,9 \mathrm{aA}$ & $1,2 \mathrm{aB}$ \\
\hline & & $\mathrm{CN}$ & $5,2 \mathrm{aA}$ & $68,9 \mathrm{aA}$ & $290,0 \mathrm{aA}$ & $4,2 \mathrm{aA}$ & $1,3 a A$ \\
\hline CV (\%) & & & 19,0 & 18,8 & 21,6 & 21,3 & 14,5 \\
\hline
\end{tabular}

* Médias seguidas das mesmas letras minúsculas, na mesma coluna, profundidade e local, não diferem entre si pelo teste de Tukey em $5 \%$ de probabilidade;

** Médias seguidas das mesmas letras maiúsculas, na mesma coluna, profundidade e cobertura, não diferem entre si pelo teste Tukey a $5 \%$ de probabilidade. ${ }^{*}$ Mean followed by same small letters, in the same column, depth and site do not differ statistically for Tukey test in $5 \%$ of probability. 
savana náo cultivada a partir de 7-8 anos do plantio (BernhardReversat, 1993).

Neste estudo, embora visualmente houvesse abundância de folhas e ramos sobre o solo nos plantios de acácia, não se estimou a entrada de liteira. Entretanto, esperar-se-ia maior aporte nos plantios de acácia em relação ao $\mathrm{CN}$ e que esta liteira tenderia a aumentar o teor de carbono orgânico no solo. De fato, houve tendência de maiores valores de $\mathrm{C}_{\mathrm{org}}$ nos plantios de acácia com valores absolutos até $16 \%$ maiores na cobertura com acácia (Tabela 2), evidenciando possíveis efeitos benéficos da introdução adicional de $\mathrm{N}$ via fixação biológica de $\mathrm{N}_{2}$ na estabilização do C do solo (Sisti et al., 2004; Resende et al., 2006; Balieiro et al., 2008). Isso indica a possibilidade de que, ao longo dos anos, deverá ocorrer aumento significativo nos estoques de carbono do solo (Bernhard-Reversat, 1993; Resh et al., 2000). Também é importante destacar que, na média geral, houve tendência de maior acúmulo do $\mathrm{C}_{\text {or }}$ em profundidade (Tabela 2), mostrando a possibilidade de incremento de carbono no perfil do solo e náo apenas em superfície.

A avaliação do C-BMS mostrou que a cobertura com acácia proporcionou valores significativamente maiores comparativamente ao $\mathrm{CN}$, e os teores de C-BMS dessa cobertura no $\mathrm{CEAB}$ foram maiores que os da fazenda Cigolina, tanto na profundidade de $0-20 \mathrm{~cm}$ como $20-40 \mathrm{~cm}$, chegando a ocorrer diferenças de mais de $45 \%$ de um local para outro (Tabela 2, Figura 1). Ao contrário, não ocorreram diferenças significativas no $\mathrm{CN}$ entre os dois locais em nenhuma das profundidades (Tabela 2).

Semelhantemente ao C-BMS, também foram observados maiores valores de RBS nos plantios de acácia em comparaçáo ao $\mathrm{CN}$ nas duas camadas de solo amostrados (Tabela 2). Entretanto, diferentemente do C-BMS, entre locais, apenas houve diferenças na cobertura com acácia na profundidade de 20-40 cm, sendo o maior valor observado no CEAB (Tabela 2).

Em relação ao $q \mathrm{CO}_{2}$, foi observado que o $\mathrm{CN}$, no $\mathrm{CEAB}$, não apresentou diferença significativa do $\mathrm{CN}$ da fazenda Cigolina, ao passo que a acácia no CEAB ocasionou reduçáo significativa do $q \mathrm{CO}_{2}$ em comparação com a fazenda Cigolina (Tabela 2, Figura 1). Na média dos dois locais, foi observado que a cobertura com acácia reduziu o $q \mathrm{CO}_{2}$ em cerca de $20 \%$, em ambas as profundidades. Esse talvez seja um indicativo de que a introdução de $\mathrm{N}$ adicional, via fixação biológica de $\mathrm{N}_{2}$ em simbiose com bactérias do grupo rizóbio, esteja sendo usado para a construçáo da biomassa microbiana e, por conseqüência, do $\mathrm{C}$ do solo.

É importante destacar que a fauna de invertebrados do solo pode apresentar papel importante na elevação do C-BMS, isto porque tem sido observado aumento na abundância destes organismos nos plantios de acácia, comparativamente à savana na África (Bernhard-Reversat, 1993). Neste caso, a liteira da acácia enriquecida em $\mathrm{N}$ seria atacada pela fauna de invertebrados e, a partir dos seus resíduos, haveria favorecimento ao desenvolvimento microbiano. Esta dinâmica também tem sido utilizada para justificar a taxa de humificaçấo mais rápida observada em plantios de acácias comparativamente com eucaliptos em solos arenosos das savanas africanas (Bernhard-Reversat, 1993).

No CN utilizado como referência, onde provavelmente existia maior estabilidade na dinâmica de carbono no solo, ou seja, equilíbrio entre a entrada e decomposição de liteira, foram constatados valores de C-BMS da ordem de 150-200 $\mathrm{mg} \mathrm{kg}^{-1}$ de solo, nos primeiros $20 \mathrm{~cm}$ de profundidade, e da ordem de 65 a $100 \mathrm{mg} \mathrm{kg}^{-1}$ na profundidade de $20-40 \mathrm{~cm}$. Com a introdução dos plantios de acácia, houve ativação da biomassa microbiana no solo, em ambas as profundidades, refletida tanto pela elevaçáo do C-BMS quanto pela RBS
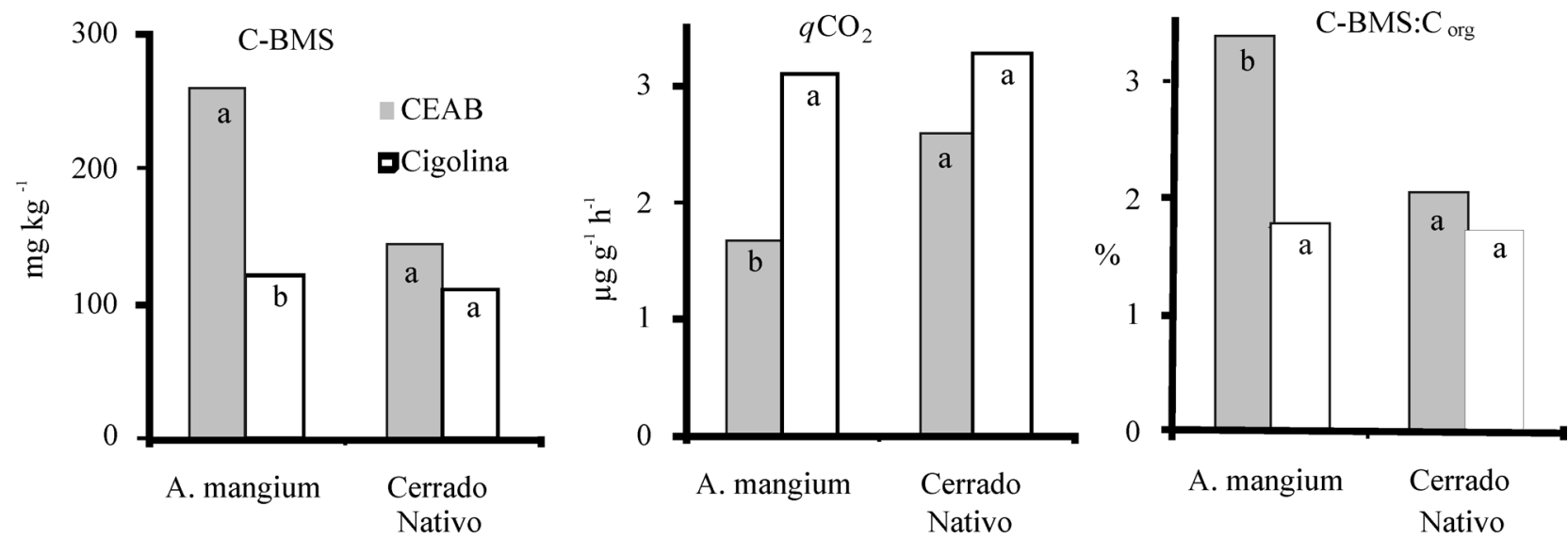

Figura 1 - Média do carbono da biomassa microbiana do solo (C-BMS), quociente metabólico $\left(q \mathrm{CO}_{2}\right)$ e relação C-BMS: $\mathrm{C}_{\text {org }}$ entre amostras de solo de plantios de Acacia mangium e Cerrado nativo de referência.

* Médias seguidas das mesmas letras para uma mesma variável e cobertura não diferem entre si pelo teste de Tukey em $5 \%$ de probabilidade. 
(Tabela 2, Figura 1). Esta ativação parece ter sido decorrente do fornecimento de substrato de melhor qualidade nutricional proporcionado pela deposição de liteira das plantas de acácia (Bernhard-Reversat, 1993; Andrade et al., 2000).

De fato, a dinâmica da biomassa microbiana do solo nas diferentes formaçôes vegetais de Roraima é ainda muito pouco conhecida, tendo sido mostrada grande flutuação dos valores desse parâmetro em um transecto floresta-savana na ilha de Maracá, ocorrendo valores na savana de até $510 \mathrm{mg}$ $\mathrm{kg}^{-1}$ C-BMS (Ross et al., 1992).

A A. mangium apresenta alta capacidade de retranslocar nutrientes das folhas senescentes para outros tecidos da planta, o que lhe fornece ampla rusticidade e potencial para ser utilizada na recuperação de áreas degradadas. Porém, isso implica em liteira com menores teores de nutrientes quando comparada à outras leguminosas (Halenda, 1989; Andrade et al., 2000). Por outro lado, como a acácia é uma leguminosa fixadora de nitrogênio, produz liteira mais enriquecida com esse nutriente quando comparada a liteira do CN que é composta, em sua grande maioria, por vegetação graminácea. Neste caso, o fornecimento de $\mathrm{N}$ teria favorecido o desenvolvimento microbiano, especialmente por este ser limitado nas savanas de Roraima.

Esta suposta melhor qualidade da liteira proporcionada pelas plantas de $A$. mangium está também refletida na relação C-BMS: $\mathrm{C}_{\text {org }}$, que representa o acúmulo de carbono nos microrganismos sem alteraçóes no estoque de carbono do solo (Gama-Rodrigues, 1997). Foi observado que a relação

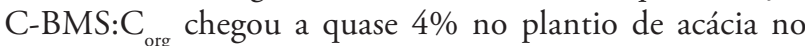
$\mathrm{CEAB}$, enquanto no $\mathrm{CN}$ este valor foi menor que 2,4 , sendo significativamente inferior (Tabela 2, Figura 1).

O C-BMS é reconhecido como um indicador de qualidade do solo capaz de mostrar as alteraçóes no ambiente em uma fase anterior às alteraçóes no teor de matéria orgânica do solo (Cattelan \& Vidor, 1990; Haynes, 2000). Desta forma, os maiores valores do C-BMS observados nos plantios de acácia, e também de menor qCO2, indicariam que a acácia estaria atuando como fonte de carbono ao solo.

Embora diferenças entre os plantios de acácia e o $\mathrm{CN}$ tenham sido observadas tanto para o C-BMS solo, RBS, $q \mathrm{CO} 2$ e a relação C-BMS:C or no $\mathrm{CEAB}$; na fazenda Cigolina elas não ocorreram (Tabela 2 ). E, além disso, estas variáveis foram também significativamente maiores no plantio de acácia do CEAB comparado ao plantio de acácia da fazenda Cigolina (Figura 1).

Descartada a possibilidade de que o efeito dos plantios de acácia nos microrganismos do solo tenha ocorrido em função da fertilidade e textura do solo, pois não foram encontradas diferenças expressivas entre os dois locais (Tabela 1), há indicativo de que a estrutura de plantio das acácias estaria interferindo na biomassa microbiana do solo.

$\mathrm{Na}$ fazenda Cigolina, estudou-se um plantio homogêneo no qual a estrutura do dossel encontrava-se fechada, com pouca luminosidade e com nenhuma entrada de liteira de outras fontes, exceto da acácia. Ao contrário, no CEAB, devido à disposição do plantio em faixas com duas linhas sendo as faixas espaçadas umas das outras por $30 \mathrm{~m}$, houve maior entrada de luminosidade e liteira de outras fontes, além da acácia. Desta forma, deve-se buscar novos trabalhos que fortaleçam ou validem as hipóteses levantadas para os mecanismos de formação de biomassa e estabilização do carbono no solo nos plantios de acácia.

O plantio de acácia no CEAB pode ter sofrido efeito de borda (Desjardins et al., 1996), no qual os fatores presentes no $\mathrm{CN}$, como a entrada de liteira, o ingresso de invertebrados do solo e a própria luminosidade atuariam dentro do plantio da acácia. Neste caso, as plantas de acácia neste local seriam meras fornecedoras de substrato para os organismos que habitam o CN.

Ao contrário, na fazenda Cigolina teria ocorrido o isolamento dos organismos do solo, previamente existente no Cerrado, além da restrição a entrada de luz e liteira de outras fontes, devido à profunda alteração do ambiente. Este isolamento teria levado à seleçáo de microrganismos menos eficientes na assimilação e utilização do carbono (Alexander, 1977; Sakamoto \& Oba, 1994).

\section{CONCLUSÕES}

1 - Os plantios de Acacia mangium com cinco anos de idade não contribuíram para o aumento do carbono orgânico do solo em área de Cerrado de Roraima;

2 - O plantio homogêneo de Acacia mangium não proporcionou alteração na biomassa microbiana do solo comparativamente ao Cerrado de referência;

3 - O plantio de Acacia mangium em fileiras duplas proporcionou aumento da biomassa microbiana do solo em relação ao plantio homogêneo e ao Cerrado de referência.

\section{AGRADECIMENTOS}

Ao pesquisador da Embrapa Roraima Dalton Roberto Schwengber pelas informaçóes sobre o plantio de $A$. mangium no Campo Experimental Água Boa - Boa Vista RR e a Empresa Ouro Verde Agrosilvopastoril Ltda. pela permissão para coleta de amostras de solo nos plantios de $A$. mangium. 


\section{BIBLIOGRAFIA CITADA}

Alexander, M. 1977. Microbial transformation of sulfur. p. 350-367. In: Alexander, M. (Ed.). Introduction to soil microbiology, 2a edição. John Wiley \& Sons, New York, NY, USA.

Anderson, T.H.; Domsch, K.H. 1990. Application of ecophysiological quotients $\left(q \mathrm{CO}_{2}\right.$ and $\left.q \mathrm{D}\right)$ on microbial biomasses from soils of different cropping histories. Soil Biology and Biochemistry, 22: 251-255.

Andrade, A.G. 1999. Ciclagem de nutrientes e arquitetura radicular de leguminosas arbóreas de interesse para revegetaçâo de solos degradados e estabilização de encostas. Tese de Doutorado, Universidade Federal Rural do Rio de Janeiro, Seropédica, Rio de Janeiro, 204pp.

Andrade, A.G.; Costa, G.S.; Faria, S.M. 2000. Deposição e decomposição da serapilheira em povoamentos de Mimosa caesalniifolia, Acacia mangium e Acacia holosericea com quatro anos de idade em Planossolo. Revista Brasileira de Ciência do Solo, 24: 777-785.

Balieiro, F.C.; Pereira, M.G.; Alves, B.J.R.; Resende, A.S.; Franco, A.A. 2008. Soil carbon and nitrogen in pasture soil aforested with eucalyptus and guachapele. Revista Brasileira de Ciência do Solo, 32: 1253-1260.

Balieiro, F.C.; Dias, F.C.; Franco, A.A.; Campello, E.F.C.; Faria, S.M. 2004. Acúmulo de nutrientes na parte aérea, na serapilhiera acumulada sobre o solo e decomposição de filódios de Acacia mangium willd. Ciência Florestal, 14: 59-65.

Bernhard-Reversat, F. 1993. Dynamics of litter and organic matter at the fast-growing tree plantations on sandy ferrallitic soils (Congo). Acta Ecologica, 14: 179-195.

Binkley, D.; Giardina, C. 1997. Nitrogen fixation in tropical forest plantations. p. 297-337. In: Nambiar, E.K.S; Brown, A.G. (Eds). Management of soil, nutrients and water in tropical plantation forests. ACAIR/CSIRO/CIFOR, Canberra, Autrália.

Campello, E.F.C. 1998. Sucessão vegetal na recuperação de áreas degradadas. p. 183-196. In: Dias, L.E.; Mello, J.W.V. (Eds.). Recuperação de Áreas Degradadas. Viçosa: Universidade Federal de Viçosa/Departamento de Solos; Sociedade Brasileira de Recuperação de Áreas Degradadas.

Cattelan, A.J.; Vidor, C. 1990. Sistemas de culturas e a população microbiana do solo. Revista Brasileira de Ciência do Solo, 14: 125-132.

Chada, S.; Campello, E.F.C.; Faria, S.M. 2004. Sucessão vegetal em uma encosta reflorestada comleguminosas. Revista Árvore, 28: 801-809.

Corazza, E.J.; Silva, J.E.; Resck, D.V.S.; Gomes, A.C. 1999. Comportamento de diferentes sistemas de manejo como fonte ou depósito de carbono em relação à vegetação de Cerrado. Revista Brasileira de Ciência do Solo, 23: 425-432.

De-Polli, H.; Guerra, J.G.M. 2008. Carbono, nitrogênio e fósforo da biomassa microbiana do solo. p. 263-276. In.: Santos, G. de A.; Silva, L.S. da; Canellas, L.P.; Camargo, F.A.O. (Eds.). Fundamentos da matéria orgânica do solo: ecossistemas tropicais \& subtropicais. $2^{\text {a }}$. edição revisada e atualizada. Metrópole, Porto Alegre, RS, Brasil.
Silva, E.E. da; Azevedo, P.H.S de; De-Polli, H. 2007. Determinação do carbono da biomassa microbiana do solo (BMS-C). Seropédica, Embrapa Agrobiologia. 6p. (Comunicado Técnico 98).

Desjardins, T.; Carneiro-Filho, A.; Mariotti, A.; Chauvel, A.; Girardin, C. 1996. Changes of the Forest-savanna boundary in Brazilian Amazonia during Holocene as revealed by soil organic carbon isotope rations. Oecologia, 108: 794-756.

Dias, L.E.; Franco, A.A.; Campello, E.F.C. 1994. Dinâmica de material orgânica e de nutrientes em solo degradado de bauxita e cultivado com Acacia mangium e Eucaliptus pellita. p. 145 153. In.: Simpósio Sul-Americano e Simpósio Nacional de Recuperação de Áreas Degradadas, 2. Foz do Iguaçu. Anais, Foz do Iguaçu.

Duguma, B.; Tonye, J.; Kanmegne J.; Manga, T.; Enoch, T. 1994. Growth of ten multipurpose tree species on acid soils in Sangmelima, Cameroon. Agroforestry Systems, 27: 107-119.

EMPRESA BRASILEIRA DE PESQUISA AGROPECUÁRIA - EMBRAPA. 2006. Centro Nacional de Pesquisa de Solos. Sistema Brasileiro de Classificação de Solos. 2 ed. Rio de Janeiro, 306 pp.

Franco, A.A.; Faria, S.M. 1997. The contribution of $\mathrm{N}_{2}$-fixing tree legumes to land reclamation and sustainability in the tropics. Soil Biology and Biochemistry, 29: 897-903.

Gama-Rodrigues, E.F. 1999. Biomassa microbiana e ciclagem de nutrientes. p. 227-240. In.: Santos, G.A.; Camargo, F.A.O. (Eds). Fundamentos da matéria orgânica do solo. Gênesis, Porto Alegre, RS, Brasil.

Garay, I.; Kindel, A.; Carneiro, R.; Franco, A.A.; Barros, E.; Abbadie, L. 2003. Comparação da matéria orgânica e de outros atributos do solo entre plantaçóes de Acacia mangium e Eucalyptus grandis. Revista Brasileira de Ciência do Solo, 27: 705-712.

Ghosh, S.; Verma, N.K. 2006. Growth and mycorrhizal dependency of Acacia mangium Willd. inoculated with three vesicular arbuscular mycorrhizal fungi in lateritic soil. New Forests, 31:75-81.

Halenda, C. 1989. Nutrient content of na Acacia mangium plantation. Nitrogen Fixing Tree Research Reports, 7: 46-48.

Haynes, R.J. 2000. Labile organic matter as an indicator of organic matter quality in arable and pastoral soils in New Zeland. Soil Biology and Biochemistry, 32: 211-219.

Jackson, L.E.; Calderon, F.J.; Steenwerth, K.L.; Scow, K.M.; Rolston, D.E. 2003. Responses of soil microbial processes and community structure to tillage events and implications for soil quality. Geoderma, 114: 305-317.

Jenkinson, D.S.; Powlson, D.S. 1976. The effects of biocida treatment on metabolism in soil - V. Method for measuring soil biomass. Soil Biology and Biochemistry, 8: 209-213

Lopes, A.S. 1984. Solos sob "cerrado": Características, propriedades e manejo. $2^{\mathrm{a}}$ ed. Potafos, Piracicaba, SP, Brasil. 162pp.

Luizão, F.J., Luizão, R.C.C. 1997. Matéria orgânica do solo em Roraima. p. 363-379. In: Barbosa, R.I.; Ferreira, E.J.G.; Castellón, E.G. (Eds). Homem, ambiente e ecologia no estado de Roraima. Instituto Nacional de Pesquisa da Amazônia (INPA). Manaus, Amazonas. 
Luizão, R.C.C.; Bonde, T.A.; Rosswall, T. 1992. Seasonal variation of soil microbial biomass-the effect of clear felling a tropical rain Forest and establishment of pasture in the Central Amazon. Soil Biology and Biochemistry, 24: 805-813.

Melo, V.F.; Gianluppi, D.; Uchoa, S.C.P. Características edafológicas dos solos do estado de Roraima. Boa Vista: Embrapa Roraima, 2003. 28p. (Embrapa Roraima. Boletim de Pesquisa e Desenvolvimento, 1)

National Research Council-N.R.C. 1983. Mangium and other fast-growing acacias for the humid tropics. Washington: National Academic Press, 65pp.

Neves, J.C.L., Gomes, J.M., Novais, R.F. 1990. Fertilização mineral de mudas de eucalipto. p. 99-126. In: Novais R.F., Barros, N.F. (Eds.). Relação-solo eucalipto. Folha de Viçosa, Viçosa, Minas Gerais.

Ngoran, A.; Zakra, N.; Ballo, K.; Kouamé, C.; Zapata, F.; Hofman, G.; Van Cleemput, O. 2006. Litter decomposition of Acacia auriculiformis Cunn. Ex Benth. and Acacia mangium Willd. under coconut trees on quaternary sandy soils in Ivory Coast. Soil Biology and Biochemstry, 43:102-106.

Remigi, P.; Faye, A.; Kane, A.; Deruaz, M.; Thioulouse, J.; Cissoko, M.; Prin, Y.; Galiana, A.; Dreyfus, B.; Duponnois, R. 2008. The exotic legume tree species Acacia holosericea alters microbial soil functionalities and the structure of the arbuscular mycorrhizal community. Applied and Environmental Microbiology, 74: 1485-1493.

Resende, A.S.; Macedo, M.; Campello, E.F.C.; Franco, A.A. 2006. Recuperação de áreas degradadas através da reengenharia ecológica. p. 315-340. In: Garay, I.; Becker, B.K. (Eds). Dimensóes Humanas da Biodiversidade. Editora Vozes. Petrópolis, Rio de Janeiro

Resh, S.C.; Binkley, D.; Parrota, J.A. 2002. Greater soil carbon sequestration under nitrogen-fixing trees compared with Eucalyptus species. Ecosystems, 5: 217-231.

Roscoe, R.; Machado, P.L.O. 2002. Fracionamento físico do solo em estudos da matéria orgânica. Embrapa Agropecuária Oestel Embrapa Solos, Dourados, MS/ Rio de Janeiro, RJ, 86pp.
Ross, S.M. 1992. Soil and litter nutrient losses in forest clearings close to forest savanna boundary on Maracá Island, Roraima. p. 145-170. In.: Furley, P.A.; Proctor, J.; Ratter, J.A. (Eds). Dynamics of forest -savanna boundaries. Champman \& Hall, London, United Kingdon.

Sakamoto, K.; Oba, Y. 1994. Effect of fungal to bacterial biomass ratio on the relationship between $\mathrm{CO} 2$ evolution and total soil microbial biomass. Biology and Fertility of Soils, 17: 39-44.

Sisti, C.P.J.; Santos, H.P.; Kohhann, R.; Alves, B.J.R.; Urquiaga, S.; Boddey, R.M. 2004. Change in carbon and nitrogen stocks in soil under 13 years of conventional or zero tillage in southern Brazil. Soil Tillage and Research, 76: 39-58.

Somdatta, G.; Verma, N.K. 2006. Growth and mycorrhizal dependency of Acacia mangium Willd. inoculated with three vesicular arbuscular mycorrhizal fungi in lateritic Soil. New Forests, 31: 75-81.

Sousa, D.M.; Lobato, E. 2004. Cerrado: Correção do solo e adubação. Embrapa Informação Tecnológica. Brasília, Distrito Federal, 416 pp.

Sposito, G.; Zabel, A. 2003. The assessment of soil quality. Geoderma, 114: 143-144.

Sun, J.S.; Simpson, R.J.; Sands, R. 1992. Nitrogenase activity of two genotypes of Acacia mangium as affected by phosphorus nutrition. Plant and Soil, 144: 51-58.

Vance, E.D.; Brookes, P.C.; Jenkinson, D.S. 1987. An extraction method for measuring soil microbial biomass C. Soil Biology and Biochemistry, 19:703-707.

Yamashita, O.S.; Hardjono, A. 2008. Soil changes induced by Acacia mangium plantation establishment: Comparison with secondary forest and Imperata cylindrical grassland soils in South Sumatra, Indonesia. Forest Ecology and Management, 254: 362-370.

Recebido em: 10/07/2008

Aceito em: 13/10/2009 